\title{
EVALUACIÓN DE GENOTIPOS MEJORADOS DE FRIJOL NEGRO Phaseolus vulgaris EN BARINAS Y MONAGAS, VENEZUELA
}

\section{EVALUATION OF IMPROVED GENOTYPES OF BLACK BEAN Phaseolus vulgaris IN BARINAS AND MONAGAS, VENEZUELA}

\author{
Águeda Duran A. ; Tania Lambert G. ${ }^{2}$; Roberto Velázquez F. ${ }^{3}$ \\ Fecha de recepción: Febrero 14 de 2014 Fecha de aceptación: Noviembre de 2014
}

\begin{abstract}
RESUMEN
La investigación se desarrolló en la finca la Alborada, sector Mamon Mamonal, municipio Rojas, en Barinas y finca La Ceiba, sector Aparicio, municipio Piar, estado Monagas en época seca en el período comprendido entre noviembre, 2011 y febrero, 2012, sobre suelos Inceptisoles y Entisoles respectivamente. El objetivo de esta investigación fue evaluar la respuesta agroproductiva de siete genotipos de frijol negro: SA 029, Línea 13, Minita, Balina, Sesentera, Silvinera y la variedad comercial Tacarigua. Se utilizó un diseño experimental de bloques al azar con arreglo bifactorial, los factores fueron la localidad y los genotipos, con siete tratamientos y cuatro repeticiones, distribuidos en 28 parcelas con un área total del experimento de $554,8 \mathrm{~m}^{2}$. El marco de siembra fue de 0,60 x 0,07 m para un total 13200 plantas totales. Se evaluaron la altura de la planta $(\mathrm{cm})$, número de vainas por planta, número de semillas por vaina, peso de 100 semillas (g) y el rendimiento $\left(\mathrm{t} . \mathrm{ha}^{-1}\right)$. Los rendimientos en el estado de Barinas fueron superiores para las variedades Balina con 1,10 t/ ha y Tacarigua con 1,06 t/ ha y en estado de Monagas, la Línea 13 con 1,92 t/ ha, seguida de la Tacarigua con 1,68 t. ha- ${ }^{-1}$ S Silvinera con 1,65 t.ha-1. El efecto económico para todas las variedades por localidad y entre ambas fue satisfactorio.
\end{abstract}

Palabras clave: Caraota negra, variedades, localidades, rendimientos, efecto económico.

1 Investigadora. M.Sc Agroecología y Desarrollo Endógeno. INIA. Barinas. Venezuela. aguedad@hotmail.com.

2 Docente. M.Sc Gestión Ambiental. Universidad de Granma. Bayamo. Cuba. tlambertg@udg.co.cu

3

Docente. Ph.D. Ciencias Agrícolas. Universidad de Granma. Bayamo. Cuba.rvelazquezf@udg.co.cu 


\begin{abstract}
The research was developed at the La Alborada farm (Mamon Mamonal sector, municipality of Rojas, state of Barinas) and La Ceibafarm (Aparicio sector, municipality of Piar, state of Monagas) on Inceptisols soils and Entisols, respectively, during dry season from November, 2011 to February, 2012.The aim of this investigation was to evaluate the agro-productive response of seven black bean genotypes: SA 029, line 13, Minita, Balina, Sesentera, Silvinera, and the Tacarigua commercial variety. A bifactorial randomized blocks design was used, the factors were the locality and the genotypes, with seven treatments and four replicas, distributed in 28 plots with a total experiment area of $554.8 \mathrm{~m}^{2}$. The sowing area was of $0.60 \times 0.07 \mathrm{~m}$ for a total of 13200 plants. Plant height $(\mathrm{cm})$, number of pods per plant, number of seeds per pod, weight of 100 seeds $(\mathrm{g})$, and yield $\left(\right.$ tha $\left.^{-1}\right)$ were evaluated. Yields in the state of Barinas were higher for the Balina and Tacarigua varieties, with $1.10\left(\right.$ t.ha $\left.^{-1}\right)$ and 1.06 t.ha $^{-1}$, respectively. In the state of Monagas, yields were higher for Line 13 variety with 1.92 t.ha ${ }^{-1}$, followed by Tacarigua with 1.68 t.ha ${ }^{-1}$, and Silvinera with 1.65 t.ha ${ }^{-1}$.The economic effect for all varieties per locality and between both localities was satisfactory.
\end{abstract}

Keywords: Black bean, varieties, localities, yields, economic effect.

\section{INTRODUCCIÓN}

El fríjol común (Phaseolus vulgaris L.) constituye la leguminosa que ha sido objeto de más estudios en América Latina, por ser la fuente principal de proteína, así como por formar parte importante de los hábitos alimentarios de la población.

En la actualidad el reconocimiento de dos grupos mayores de germoplasma de fríjol (mesoamericano y andino) ha ayudado a confirmar su participación en la selección y desarrollo de los cultivares mejorados que dominan la preferencia de los agricultores empresariales y consumidores (Voysest, 2000). El conocimiento de la diversidad genética puede ayudar a generar acciones para su conservación, uso eficiente y mejoramiento (Singh, 2001). Este conocimiento incluye las características morfoagronómicas, bioquímicas, genéticas y otras, en el nivel de la semilla y de la planta (Singh, 2001; Voysest, 2000).

La mayoría de los agricultores de Venezuela, específicamente en las localidades de Barinas y Monagas, no utilizan variedades mejoradas y en el mejor de los casos mantienen sus materiales locales. Entre las razones que podrían explicar la baja adopción de nuevas variedades están: el no haber tenido acceso a nuevos materiales, el que éstas no reunieran los atributos requeridos para las condiciones agroecológicas y socioeconómicas, o quizás por razones económicas o culturales (Morros, 2001).

Las razones antes expuestas y la necesidad de contribuir con el desarrollo de los programas alimentarios y disminuir la vulnerabilidad alimentaria, y teniendo como problema científico enfunción del desarrollo endógeno delocalidades dedicadas a este cultivo, la poca existencia de variedades mejoradas y locales, con adecuados niveles de adaptación que incrementen el rendimiento agrícola, se planteó la siguiente hipótesis de trabajo; mediante la introducción de genotipos mejorados y de procedencia local prospectados de caraota negra, se pueden lograr 
incrementos en los rendimientos, calidad del cultivo y disponibilidad de semilla, en áreas dedicadas a este cultivo. Partiendo del problema científico y la hipótesis referida, el trabajo tuvo como objetivo evaluar respuesta agro productiva de siete genotipos mejorados de caraota negra en dos localidades agroecológicas de Monagas y Barinas, Venezuela.

\section{MATERIALES Y MÉTODOS}

\section{Época de siembra, ubicación y caracterización edafoclimática de las áreas experimentales}

La investigación se desarrolló en dos Estados, Barina y Monagas en la época seca, en el período comprendido desde el 13 de diciembre de 2011 hasta el 26 de febrero del 2012, en la finca la Alborada, sector Mamon Mamonal, municipio Rojas, ubicada a los $20^{\circ} 20^{\prime} 00^{\prime \prime}$ de latitud norte y $76^{\circ} 41^{\prime} 00^{\prime \prime}$ de longitud oeste; a una altitud de 114 msnm y en el período comprendido desde el 6 de noviembre del 2011, hasta el 26 de febrero del 2012, en la finca La Ceiba, sector Aparicio, municipio Piar ubicada a los $10^{\circ} 00^{\prime} 36^{\prime \prime}$ de latitud norte y $63^{\circ} 34^{\prime} 12^{\prime \prime}$ de longitud oeste a una altitud de $307 \mathrm{msnm}$, respectivamente.

\section{Muestreo y métodos de análisis agroquímico de los suelos}

Las muestras de suelo de las áreas experimentales se tomaron a la profundidad de $20 \mathrm{~cm}$ para análisis químicos y físicos, realizados en el Laboratorio de Análisis y Procesamiento de Suelos de UNELLEZ - Guanare y de INIA - Monagas. Los datos obtenidos permiten caracterizar a los mismos acorde con su fertilidad y aptitud para el cultivo de la caraota (Tab.1).
Tabla 1. Métodos de análisis y procesamiento de suelos utilizado en el laboratorio -UNELLEZ Guanare, estado Portuguesa e INIA para las muestras de suelo de dos localidades

\begin{tabular}{l|l}
\hline Análisis & Métodos \\
\hline Materia orgánica & Walkey Black \\
\hline Sales solubles & Conductividad eléctrica \\
\hline Fósforo & Olsen \\
\hline Potasio & $\begin{array}{l}\text { Acetato de Amonio - } \\
\text { fotometría de llama }\end{array}$ \\
\hline Calcio y magnesio & Acetato de amonio \\
\hline $\mathrm{pH}$ & Peachímetro \\
\hline
\end{tabular}

\section{Características climáticas de las localidades estudiadas}

Las características climáticas de ambas regiones de estudio (datos mensuales de precipitación en $\mathrm{mm}$, temperatura media en ${ }^{\circ} \mathrm{C}$ y de humedad relativa en \%) fueron tomadas de la red agrometeorológica del INIA para los años 1998 al 2011, histórica de datos climáticos (INIA, 2013).

\section{Manejo de cultivo}

La preparación del terreno se efectuó con cuatro pases cruzados de rastra, se aplicó fertilización básica con fórmula completa N - P - K (10-2020), a razón de $200 \mathrm{~kg} /$ ha y tres aplicaciones de abono foliar durante el ciclo del cultivo en dosis de 3 1/ha; se realizó un adecuado control de plagas con cipermetrina (perforadores y masticadores) a razón de $300 \mathrm{cc} /$ ha y no fue necesario dar riego por las precipitaciones ocurridas durante el período.

Las malezas fueron controladas mediante la aplicación del herbicida pre emergente pendimetalin en dosis de 4 l/ha, posteriormente se dieron dos limpias manuales. 
La cosecha se realizó manualmente a los 75 días luego de la emergencia del cultivo, al ocurrir el cambio de coloración de las vainas y cuando las plantas habían perdido casi la totalidad de las hojas.

\section{Diseño experimental y tratamientos}

Se utilizó un diseño de bloque al azar, con arreglo bifactorial con 7 tratamientos y 4 réplicas, cada tratamiento tenía una parcela de cuatro hileras de seis metros de largo y 1,8 de ancho, distribuidos en 32 parcelas de $6 \mathrm{~m}$ de largo y 1,8 $\mathrm{m}$ de ancho, para un área de parcela de 10,8 $\mathrm{m}^{2}$. La distancia entre bloques de $2 \mathrm{~m}$, para un área total del experimento de $554,8 \mathrm{~m}^{2}$. La siembra se realizó a una profundidad de $2 \mathrm{~cm}$ según lo establecido por las normas del cultivo (SENASEM, 2010) para un total de 257 plantas/ parcela y 13202 plantas totales empleando semillas de siete variedades utilizadas como tratamientos de caraotas negras (Tab. 2). Las semillas utilizadas fueron de alta calidad, procedentes del banco de germosplasma del INIA.

\section{Datos del pasaporte de las variedades utiliza- das en la investigación}

Para la realización de la investigación, se evaluaron siete variedades de caraota negra, tres pertenecen al programa de mejoramiento genético del Instituto Nacional de Investigaciones Agrícolas (INIA) y cuatro desarrollados por los agricultores.

Del total de las variedades dos son experimentales, cuatro locales y una comercial. A continuación (Tab. 2), se exponen los datos del pasaporte de las variedades utilizadas en los experimentos.

\section{Variables evaluadas:}

Para llevar a cabo la medición de las diferentes variables se tomaron de los dos surcos centrales de cada parcela, 10 plantas al azar en cada réplica, para un total de cuarenta por tratamiento. Las variables evaluadas fueron:

Días hasta la floración: El número de días transcurrido desde la siembra en suelo húmedo hasta que el $50 \%$ de las plantas en la parcela presentaron el $50 \%$ de floración.

Hábito de crecimiento: Determinado en la etapa de floración y madurez fisiológica del cultivo, clasificando los genotipos en cuatro tipos: I Arbustivo determinado, II Arbustivo indeterminado, III Postrado indeterminado y IV Trepador indeterminado.

Altura de la planta $(\mathrm{cm})$ : Se realizó la medición desde el punto de inserción de las raíces hasta

Tabla 2. Datos del pasaporte de las variedades utilizadas en la investigación

\begin{tabular}{l|l|l|l|l}
\hline $\mathrm{N}^{\circ}$ & \multicolumn{1}{|c|}{ Nombre } & \multicolumn{1}{c|}{ Certificada } & \multicolumn{1}{c}{ Procedencia } & \multicolumn{1}{c}{$\begin{array}{c}\text { Tipo de } \\
\text { crecimiento }\end{array}$} \\
\hline 1 & Tacarigua & Comercial & INIA & Indeterminado \\
\hline 2 & SA O29 & Experimental & INIA & Indeterminado \\
\hline 3 & Línea 13 & Experimental & INIA & Indeterminado \\
\hline 4 & Minita & Local de Aragua & Agricultor estado Aragua & Indeterminado \\
\hline 5 & Balina & Local de Portuguesa & Agricultor estado Portuguesa & Indeterminado \\
\hline 6 & Sesentera & Local de Lara & Agricultor estado Lara & Indeterminado \\
\hline 7 & Silvinera & Local de Lara & Agricultor estado Lara & Indeterminado \\
\hline
\end{tabular}


el último meristemo apical del tallo empleando una cinta métrica. Al final de la floración.

Número de vainas por planta: Se contaron las vainas que tuvieron por lo menos una semilla viable en cada planta.

Número de semillas por vaina: Se contó el número de semillas viables de cada una de las vainas cosechada.

Peso de 100 semillas (g): De acuerdo a su peso se clasificaron en tres grupos de tamaño de semilla, según el sistema estándar para la evaluación de germoplasma de fríjol: Se clasifica según el modelo siguiente:

$1=$ Semilla pequeña, si su peso es menor de $25 \mathrm{~g}$ 2= Semilla mediana, si su peso está entre 25 y $40 \mathrm{~g}$ $3=$ Semilla grande, si su peso es mayor de $40 \mathrm{~g}$

Rendimiento (t.ha-1): Se determinó a partir de la producción obtenida en las 10 plantas seleccionadas y el área experimental evaluada, luego se transformó a t.ha-1.

\section{Análisis estadísticos}

Los resultados de la investigación para los experimentos fueron sometidos a un análisis de varianza de clasificación doble. En las variables que hubo diferencias significativas entre las medias de tratamientos, se utilizó como criterio discriminante la prueba de Duncan para un nivel de error del $5 \%$ para la comparación múltiple de medias (Snedecor et al., 1984), empleando el paquete estadístico InfoStat, versión 1.1 (2002).

\section{Indicadores económicos de los resultados}

La valoración económica de los resultados en cada experimento, se realizó según la metodología propuesta por la FAO (1980), evaluando los siguientes indicadores:

Valor de la producción (Bs.): Rendimiento promedios de las variedades multiplicado por el precio de venta de una tonelada de producto de semilla.

$\mathrm{Vp}=\mathrm{PB} \times \mathrm{PV}$ donde:

VP: Valor de la producción

PB: A $\times$ R

PB: Producción bruta $(\mathrm{kg} / \mathrm{ha})$

A: Área (ha)

$\mathbf{R}$ : Rendimiento $\mathrm{kg}$

PV: Precio de compra semilla (Bs)

Costo de producción (Bs/ha): Gastos incurridos para la producción de una hectárea de caraota.

$\mathbf{C P}=\sum \mathrm{Gp}$ donde

Cp: Costo directos para la producción de semilla de caraota (Bs).

GP: Gastos de producción directo

Beneficio (Bs.): Ganancia neta obtenida por diferencia neta entre el valor de la producción y el costo de producción.

$\mathrm{G}=\mathrm{Vp}-\mathrm{Cp}$

Vp: valor de la producción (Bs)

Cp: Costo de producción (Bs/ha)

\section{Rentabilidad:}

$$
\operatorname{Re}=\mathrm{U} / \mathrm{Cp} X 100
$$

Re: Rentabilidad (\%)

$\mathrm{U}$ : utilidad (Bs).

Cp: costo de producción (Bs/ha)

Para el cálculo de estos indicadores, se utilizó como información básica: 
1. Costo real de producción de una hectárea de semilla: 4.660,87 Bs/ha

2. Precio de caraota de semilla (Bs/t): 9000, según punto de cuenta del INIA

\section{RESULTADOS Y DISCUSIÓN}

\section{Características edáficas y climáticas de las re- giones de estudio}

El experimento se estableció en Barinas sobre un suelo, que según la clasificación de los suelos de Venezuela, pertenece a los inceptisoles (Schargel, 1972) y Monagas sobre un suelo, que según la clasificación de los suelos pertenece a los entisoles (Zinck y Urriola, 1971) (Tab. 3).

El análisis agroquímico para el suelo de Barinas confirma, que es un suelo con fertilidad caracterizada por contenido alto de fósforo y calcio, medio de potasio y magnesio y bajo de materia orgánica, con un $\mathrm{pH}$ ligeramente alcalino, textura media. Para el suelo de Monagas indica que es un suelo caracterizado por un contenido bajo de fósforo, potasio y calcio y alto en materia orgánica, y magnesio, con un $\mathrm{pH}$ neutro, textura liviana. Ambos se evalúan como suelos aptos para la producción de leguminosas (Morros, 2001).

\section{Precipitaciones}

Para ambas localidades, las precipitaciones alcanzaron valores promedios mensuales entre 7 y $145 \mathrm{~mm}$, las cuales son relativamente bajas. Según Bastidas (1974), las caraota se desarrollan bien con lluvias de 300 a $400 \mathrm{~mm}$ anuales mínimas, por lo que se recomienda sembrar al final de la estación de lluvias, cuando éstas son menos intensas, pero suficientes para cubrir las necesidades de agua del cultivo y que la cosecha coincida con la época seca.

\section{Temperatura}

En cuanto a las temperaturas promedios mensuales, estas entre los meses de diciembre a febrero, fueron de 26 a $27^{\circ} \mathrm{C}$ en Barinas y dada la mayor altitud, de 19 a $26^{\circ} \mathrm{C}$ en Monagas. Para ambos casos, las temperaturas medias mensuales, mostraron valores que oscilaron entre los 20 a $26^{\circ} \mathrm{C}$, las que se ajustan al cultivo de la caraota. En cuanto a la temperatura, la planta de caraota crece bien entre temperaturas promedio de $15 \mathrm{a}$ $27^{\circ} \mathrm{C}$, aunque hay un gran rango de tolerancia entre las distintas variedades cultivadas. En términos generales, bajas temperaturas retardan el crecimiento, mientras que altas temperaturas lo aceleran. Los extremos pueden producir problemas, por ejemplo: falta de floración o esterilidad (Benacchio, 1982).

Tabla 3. Propiedades químicas y físicas de los suelos de Barinas y Monagas

\begin{tabular}{l|l|c|c|c|c|c|c}
\hline Localidad & Textura & $\begin{array}{c}\text { Materia } \\
\text { orgánica } \\
(\%)\end{array}$ & $\begin{array}{c}\text { Fósforo } \\
(\mathrm{ppm})\end{array}$ & $\begin{array}{c}\text { Potasio } \\
(\mathrm{ppm})\end{array}$ & $\begin{array}{c}\text { Calcio } \\
(\mathrm{ppm})\end{array}$ & $\begin{array}{c}\text { Magnesio } \\
(\mathrm{ppm})\end{array}$ & $\begin{array}{c}\mathrm{pH} \\
\text { (Rel:1:2) }\end{array}$ \\
\hline Barinas & $\begin{array}{l}\text { Arcillo } \\
\text { Limoso }\end{array}$ & 2,79 & 22 & 150 & 1750 & 250 & 7,5 \\
\hline Monagas & $\begin{array}{l}\text { Franco } \\
\text { arenoso }\end{array}$ & 5,132 & 12 & 70 & 1011 & 818 & 7 \\
\hline
\end{tabular}




\section{Humedad relativa}

Para la humedad relativa en ambas localidades, se presentaron valores entre el 64 al $85 \%$, característico de una atmósfera moderadamente seca a húmeda, las cuales son adecuadas para el cultivo de la caraota, correspondiéndose con lo planteado por (Bastidas, 1974), el cual expresa que el fríjol caraota se adapta muy bien a zonas de clima cálido y cálido moderado con humedades relativas medias.

Según Morro (2001), la caraota es una planta de días cortos, pero presenta una amplia adaptación con respecto a la altitud. Se consiguen siembras entre los 150 y 2.000 msnm. Benacchio (1982) cita como la altitud óptima del cultivo entre 500 y $1.000 \mathrm{msnm}$. La cantidad de agua requerida por la caraota oscila entre los 300 y $500 \mathrm{~mm}$; esto nos indica que es un cultivo poco exigente en cuanto a cantidad, pero si necesita una adecuada distribución del agua durante su ciclo.

Evaluación de la respuesta agroproductiva de siete genotipos de caraota negra en dos localidades de Venezuela

\section{Número de días hasta la floración}

Los resultados de estas variables fueron muy similares para todos los genotipos en sus diferentes repeticiones. En la Tabla 4 se aprecia, que la floración en casi todas las variables se encuentra entre los 36 a 38 días, lo que coincide con resultados similares obtenidos por (Valderrama et al., 1997) entre las variedades más tempranas investigadas hasta inicios de la floración.

Para ambas localidades, mostraron mayor estabilidad genética las variedades SA029, Línea 13 y Sesentera. Esta autora observó, que a menor altura sobre el nivel del mar (155 msnm), las variedades tienden a ser más precoces, probablemente por mayor temperatura promedio, lo que coincide con nuestros resultados, donde altura de 114 msnm para Barinas, contra 307 para Monagas, marcan la escasa diferencia entre ambos ecosistemas a favor de la precocidad en Barinas.

Tabla 4. Días a la floración de los genotipos estudiados

\begin{tabular}{l|c|c}
\hline \multirow{2}{*}{ Tratamientos } & \multicolumn{2}{|c}{ Días de la floración } \\
\cline { 2 - 3 } & \multicolumn{2}{|c}{ Localidades } \\
\cline { 2 - 3 } & Barinas & Monagas \\
\hline Tacarigua & 37 & 36 \\
\hline SA 029 & 36 & 36 \\
\hline Línea 13 & 37 & 37 \\
\hline Minita & 36 & 37 \\
\hline Balina & 36 & 38 \\
\hline Sesentera & 36 & 36 \\
\hline Silvinera & 36 & 38 \\
\hline
\end{tabular}

\section{Hábito de crecimiento y altura de la planta}

Estudios hechos por el programa de fríjol en el CIAT (1984), se consideró que los hábitos de crecimiento podrían ser agrupados en cuatro tipos principales: Tipo I, determinado arbustivo; Tipo II, indeterminado arbustivo; Tipo III, indeterminado postrado; Tipo IV, indeterminado trepador (Debouck e Hidalgo, 1985).

Los genotipos Tacarigua, SA 029, Línea 13, Minita, Balina y Silvinera, tuvieron un hábito de crecimiento de arbusto indeterminado sin guía (CIAT, 1984), mientras que el Sesentera fue postrado indeterminado con guía trepadora (Tab. 5). La importancia de este indicador se relaciona con la preferencia del agricultor de trabajar con variedades de crecimiento arbustivo indeterminado (tipo II), lo que se alcanza en las variedades objeto de introducción. 
Al analizar la variable altura de la planta (Tab.5), para ambas localidades resultó significativo el efecto genotipo, localidad e interacción genotipo por ambiente, siendo en Monagas donde las plantas dieron una mejor respuesta a esta variable y en ambas localidades la Sesentera para la altura de la planta. Los genotipos Sesentera y Balina alcanzaron un valor promedio de 75, 93 cm y 72, $48 \mathrm{~cm}$ respectivamente, sin diferencias desde el punto de vista estadístico, entre ellas, y sí entre Sesentera y el resto de los genotipos. El valor más bajo lo obtuvo el Tacarigua con altura con un promedio de $54,43 \mathrm{~cm}$. En Barinas coincidió que la Sesentera obtuvo los mayores valores promedios $(70,36 \mathrm{~cm})$ y Tacarigua el más bajo $(45,48 \mathrm{~cm})$.

Tabla 5. Hábito de crecimiento y altura de la planta en las en las localidades de Barinas y Monagas

\begin{tabular}{|c|c|c|c|c|}
\hline \multirow{3}{*}{ Genotipo } & \multicolumn{2}{|c|}{ Hábito de crecimiento } & \multicolumn{2}{|c|}{ A.P/Localidad } \\
\hline & \multicolumn{4}{|c|}{ Localidades } \\
\hline & Barinas & Monagas & Barinas & Monagas \\
\hline Tacarigua & Arbusto indeterminado sin guía & Arbusto indeterminado sin guía & $45,48 \mathrm{~g}$ & $54,43 \mathrm{f}$ \\
\hline SA 029 & Arbusto indeterminado sin guía & Arbusto indeterminado sin guía & $47,23 \mathrm{~g}$ & 65,50 cde \\
\hline Línea 13 & Arbusto indeterminado sin guía & Arbusto indeterminado sin guía & 64,68 de & $69,20 \mathrm{bcd}$ \\
\hline Minita & Arbusto indeterminado sin guía & Arbusto indeterminado sin guía & $55,25 \mathrm{f}$ & 64,40 de \\
\hline Balina & Arbusto indeterminado sin guía & Arbusto indeterminado sin guía & $69,28 \mathrm{bcd}$ & $72,48 \mathrm{ab}$ \\
\hline Sesentera & $\begin{array}{l}\text { Postrado indeterminado con } \\
\text { guía trepadora }\end{array}$ & $\begin{array}{l}\text { Postrado indeterminado con guía } \\
\text { trepadora }\end{array}$ & $70,36 \mathrm{bc}$ & 75,93 a \\
\hline Silvinera & Arbusto indeterminado sin guía & Arbusto indeterminado sin guía & $61,43 \mathrm{e}$ & 64,30 de \\
\hline & & & $\mathrm{CV}=5,71$ & $\mathrm{CV}=5,71$ \\
\hline & & & $\mathrm{R}^{2}=0,89$ & $\mathrm{R}^{2}=0,89$ \\
\hline
\end{tabular}

Promedio con columnas con letras distintas indican diferencias significativas $(p \leq 0,05)$

La respuesta de los genotipos en Monagas quizás fue debido a que los suelos de esta localidad tienen mayor contenido de materia orgánica con una textura liviana, mientras que el de Barinas tienen bajo contenido de materia orgánica con una textura media lo que facilitó mayor desarrollo de las plantas, aspectos coincidentes con lo analizado en epígrafes anteriores, en este sentido se plantea, que la caraota prefiere suelos livianos, con buena fertilidad y un subsuelo permeable para evitar encharcamiento donde el pH óptimo está entre
5,5 y 7,0; fuera de este rango deben hacerse algunas correcciones.

Estos resultados superan a los obtenidos por Lozada et al. (1983), en estudio de 14 variedades de caraota en el Estado de Lara, obteniendo una altura promedio $44 \mathrm{~cm}$, superan además a los obtenidos por Najul y Anzalones.(2006), en estudios realizados en control de malezas con cobertura en caraota en condiciones del estado Lara, donde las plantas alcanzaron una altura promedio entre 42,75 y $51,85 \mathrm{~cm}$. 
Vainas por planta, semillas por vainas y peso de 100 semillas

Para la variable vainas por planta (Tab. 6), la Sesentera alcanzó los mejores resultados con una cantidad promedio de 41,86 , difiriendo significativamente del resto de los genotipos, siendo Tacarigua la de menor cantidad de vainas $(20,25)$. De forma general el genotipo Sesentera para ambas localidades y para ambas variables fue quien respondió con los valores más altos y la Tacarigua con los valores más bajos.

Valderrama et al. (1997), en Colombia, obtuvieron un promedio de vainas por planta para diferentes genotipos entre 13,25 (MCR 1020) y 35 (MCR 1011), el testigo local se ubicó en la posición quinta con 22 vainas/planta, y el testigo internacional ocupó la posición 19 con 16,58 vainas/ planta. Evaluaciones realizadas por Bastidas y Camacho (1969) en la variedad ICA-Tui, reportan para esta variable en Palmira en tres semestres, un promedio de 17,7 , valor que fue superado por muchos de los genotipos evaluados en Arauquita. Estos resultados de ambos autores, son similares a los alcanzados para Barinas e inferiores para Monagas, lo que indica una mayor producción de vainas por planta a favor de esta última localidad.

El número de vainas por plantas de la Sesentera obtenida en la localidad de Monagas superó a los resultados obtenidos por Valderrama et al. (1997), en evaluación de 26 genotipos de caraota en la zona de Vega del Río Arauca con resultados que oscilaron entre 14 y 3 , superan también a los resultados obtenidos por Figueroa y Warnock (2001).

En la Tabla 6 se muestran también los resultados del análisis del número de semillas por vaina, observándose que no resultó significativo el efecto localidad, ni la interacción genotipo $x$ ambiente. Entre las variables se presentó diferencias significativas entre SA 029 con respecto a los demás genotipos, la cual alcanzó el valor más bajo con una cantidad promedio de 3,88 semillas por vaina, mientras que entre el resto no existió diferencias.

Tabla 6. Vainas por planta, semillas por vainas y peso de 100 semillas de las dos localidades

\begin{tabular}{|c|c|c|c|c|c|}
\hline \multirow{3}{*}{ Genotipo } & \multicolumn{2}{|c|}{ V.P/Localidad } & \multirow{3}{*}{$\begin{array}{l}\text { Semillas por } \\
\text { vainas }\end{array}$} & \multirow{2}{*}{\multicolumn{2}{|c|}{$\begin{array}{l}\text { Peso de } 100 \text { semillas }(\mathrm{g}) / \\
\text { Localidad }\end{array}$}} \\
\hline & \multirow{2}{*}{ Barinas } & \multirow{2}{*}{ Monagas } & & & \\
\hline & & & & Barinas & Monagas \\
\hline Tacarigua & $16,38 \mathrm{~d}$ & $20,25 \mathrm{~cd}$ & $5 a$ & $16,15 \mathrm{~cd}$ & $23,87 \mathrm{~b}$ \\
\hline SA 029 & $16,43 \mathrm{~d}$ & $26,75 \mathrm{~b}$ & $3,88 \mathrm{~b}$ & $14,87 \mathrm{~cd}$ & $24,46 \mathrm{~b}$ \\
\hline Línea 13 & $17,38 \mathrm{~d}$ & $28,25 \mathrm{~b}$ & $4,75 \mathrm{a}$ & $16,30 \mathrm{~cd}$ & $28,90 \mathrm{a}$ \\
\hline Minita & $16,63 \mathrm{~d}$ & $24,75 \mathrm{bc}$ & $4,75 \mathrm{a}$ & $14,10 \mathrm{~d}$ & $22,78 \mathrm{~b}$ \\
\hline Balina & $16,30 \mathrm{~d}$ & $28,25 \mathrm{~b}$ & $4,63 \mathrm{a}$ & $17,65 \mathrm{c}$ & $23,23 \mathrm{~b}$ \\
\hline Sesentera & $14,72 \mathrm{~d}$ & $41,86 \mathrm{a}$ & $4,58 \mathrm{a}$ & $17,44 \mathrm{c}$ & $24,13 \mathrm{~b}$ \\
\hline Silvinera & $15,60 \mathrm{~d}$ & $23,80 \mathrm{bc}$ & $4,83 \mathrm{a}$ & $17,47 \mathrm{~cd}$ & $24,17 \mathrm{~b}$ \\
\hline \multicolumn{3}{|l|}{$C V=16,64$} & $\mathrm{CV}=12,80$ & \multicolumn{2}{|l|}{$C V=9,76$} \\
\hline \multicolumn{3}{|l|}{$\mathrm{R}^{2}=0,88$} & $\mathrm{R}^{2}=0,35$ & \multicolumn{2}{|l|}{$\mathrm{R}^{2}=0,87$} \\
\hline
\end{tabular}

Promedio con columnas con letras distintas indican diferencias significativas $(p \leq 0,05)$ 
Los resultados obtenidos en el número de granos son similares a los obtenidos por Valderrama et al. (1997), donde obtuvieron valores entre 3,43 y 5 semillas por vainas. (Barrios y Ortega, 1968) reportaron que la variedad Tacarigua bien desarrollada posee de seis a siete granos y en raras ocasiones puede llegar hasta ocho.

Con respecto al peso de 100 semillas, resultó significativo el efecto del genotipo, localidad y la interacción genotipo por localidad, siendo en Monagas donde se alcanzaron los mayores valores, el genotipo Línea 13 fue el de mejor respuesta, alcanzando un peso promedio de 28,90 $\mathrm{g}$, difiriendo significativamente con el resto de los genotipos, sin embargo todos los demás genotipos se comportaron de forma similar sin diferencias entre ellos.

El peso de 100 semillas en la localidad de Monagas, superó a los de Barinas de forma significativa para todas las variedades y son a la vez superiores a los alcanzados por autores como Barrios y Ortega (1968) y Ismael et al. (2011) y para ambas localidades está en correspondencia a los obtenidos por Valderrama et al. (1997), donde el valor promedio osciló entre 14 y 28 g.

\section{Rendimientos}

Los resultados expuestos muestran (Fig. 1) que en el estado de Barinas las variedades con mayor rendimiento y que no difieren significativamente entre ellas son Balina con 1.10 t.ha $^{-1} \mathrm{y}$ Tacarigua con 1,06 t.ha-1. Para el estado de Monagas la variedad Línea 13 fue la de mejores resultados con 1,92 t.ha-1 ${ }^{-1}$ que no mostró a la vez diferencias significativas con las variedades Tacarigua con 1,68 t.ha-1 y Silvinera con 1,65 t.ha-1 . Resultó significativo el efecto genotipo y la localidad, donde SA 029 obtuvo el valor más bajo $\left(0,99\right.$ t.ha $\left.^{-1}\right)$ existiendo diferencias significativas entre él y el resto de los genotipos.

Se puede inferir que los resultados pudieron estar influenciados por las condiciones edafoclimáticas de las localidades, las cuales fueron favorables para el desarrollo del cultivo, especialmente en Monagas. (Benacchio, 1982), cita que la cantidad de agua requerida por la caraota oscila entre los 300 y $500 \mathrm{~mm}$ y (Rodríguez et al., 1995) señalan que la caraota tampoco tolera excesos de agua y la temperatura óptima oscila entre 15 a $27^{\circ} \mathrm{C}$, aunque hay un gran rango de tolerancia entre las distintas variedades cultivadas.

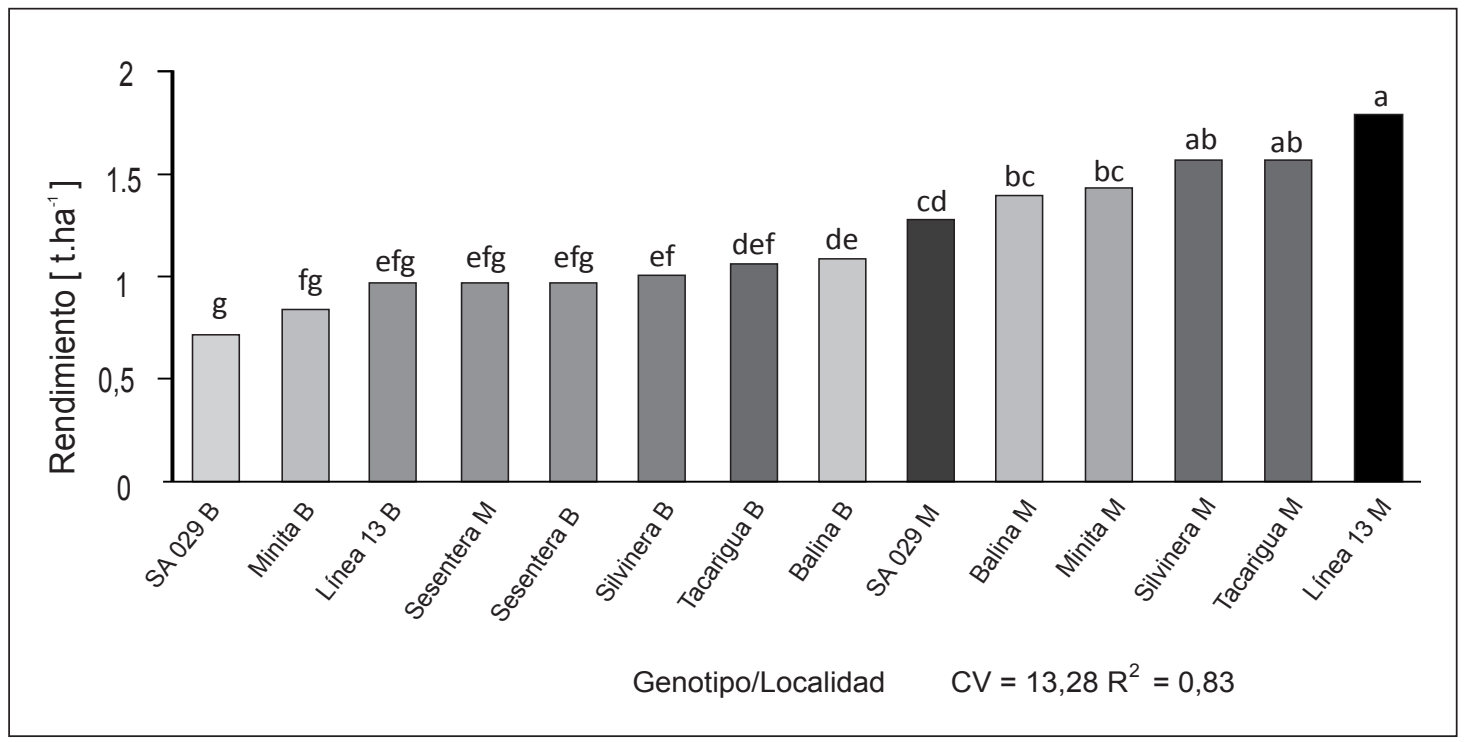

Figura 1. Rendimientos en t.ha ${ }^{-1}$ de ambas localidades 
Los rendimientos obtenidos son similares a los logrados por (INTA, 2004) y con respecto a la variedad Tacarigua que es la única comercial, están es correspondencia con los resultados históricos en Venezuela que oscilan entre 1,3 y 1,6 t.ha ${ }^{-1}$ (Ortega y Barrios, 2002).

\section{Valoración económica}

Se pudo observar que todos los indicadores económicos mostraron buenos resultados (Tab 7). El efecto económico para todas las variedades por localidad y entre ambas fueron satisfactorios, las mayores utilidades para todas las variedades ocurrieron en la localidad de Monagas, lo que se corresponde con los rendimientos superiores obtenidos, dadas las mejores condiciones para el desarrollo del cultivo, y para esta misma localidad, las variedades Línea 13 y Tacarigua superaron en ganancias a las restantes. La mayor estabilidad con base en las utilidades obtenidas para ambas regiones, fue alcanzada por la variedad Tacarigua.

El valor de la producción está directamente relacionado con el rendimiento de las variedades y el precio de compra, que para este caso de tomo como constante $(9.000 \mathrm{Bs}$./T). Teniendo en cuenta esto, ninguno de los rendimientos de la localidad de Barinas, supera al peor rendimiento de la localidad de Monagas, por lo tanto al estar relacionado el rendimiento con el valor de la producción con precio de compra fijo, los más altos valores de producción se dan en la localidad de Monagas, con las variedades: Línea 13 en 17,280.oo Bs/Ha, Tacarigua con 15,120 Bs/Ha. y Silvinera con 14,850.oo Bs/Ha. Por lo tanto los menores ingresos se encuentran en la localidad de Barinas, siendo el de la variedad SA 029 la más baja con 6,570.oo Bs/Ha.

Los costos de producción se tomaron constantes para las dos localidades en 4,660.87 Bs/Ha. Los cálculos de la utilidad (Bs/Ha.), resultantes de la diferencia entre el valor de la producción y los costos directos de producción fueron más altos en la localidad de Monagas y más bajos en Barinas. La utilidad más alta se refleja en la variedad Línea 13 de la Localidad de Monagas con 10,787.86 Bs/Ha. y, la más baja en Barinas con la variedad SA 029 en 1,909.13 Bs/Ha.

En el análisis de rentabilidad podemos afirmar que las variedades de Monagas superan el $100 \%$, a excepción de SA 029 que obtuvo una rentabilidad del $87 \%$ y, esta misma variedad en Barinas obtuvo la rentabilidad más baja con un $41 \%$.

Tabla 7. Valoración económica de las localidades Barinas y Monagas en Bolívares

\begin{tabular}{l|c|c|c|c|c|c|c|c|c|c}
\hline \multirow{2}{*}{ Tratamientos } & \multicolumn{9}{|c}{ Indicadores económicos } \\
\cline { 2 - 12 } & \multicolumn{2}{|c|}{ Rend. (t.ha-1) } & \multicolumn{2}{|c}{ V/P } & \multicolumn{2}{c}{ CP (Bs/ha) } & \multicolumn{2}{c}{ U (Bs/ha) } & \multicolumn{3}{c}{$\operatorname{Re~(\% )~}$} \\
\cline { 2 - 12 } & Barinas & Monagas & Barinas & Monagas & Barinas & Monagas & Barinas & Monagas & Barinas & Monagas \\
\hline Tacarigua & 1,06 & 1,68 & $9.540,00$ & $15,120,00$ & $4,660,87$ & $6,492,14$ & $4,879,13$ & $8,627,86$ & 105 & 133 \\
\hline SA 029 & 0,73 & 1,35 & $6.570,00$ & $12,150,00$ & $4,660,87$ & $6,492,14$ & $1,909,13$ & $5,657,86$ & 41 & 87 \\
\hline Línea 13 & 0,96 & 1,92 & $8.640,00$ & $17,280,00$ & $4,660,87$ & $6,492,14$ & $3,979,13$ & $10,787,86$ & 85 & 166 \\
\hline Minita & 0,83 & 1,49 & $7.470,00$ & $13,410,00$ & $4.660,87$ & $6,492,14$ & $2,809,13$ & $6,917,86$ & 60 & 107 \\
\hline Balina & 1,10 & 1,43 & $9.900,00$ & $12,870,00$ & $4,660,87$ & $6,492,14$ & $5,239,13$ & $6,377,86$ & 112 & 98 \\
\hline Sesentera & 0,98 & 1,57 & $8.820,00$ & $14,130,00$ & $4,660,87$ & $6,492,14$ & $4,159,13$ & $7,637,86$ & 89 & 118 \\
\hline Silvinera & 1,02 & 1,65 & $9.180,00$ & $14,850,00$ & $4,660,87$ & $6,492,14$ & $4,519,13$ & $8,357,86$ & 97 & 129 \\
\hline
\end{tabular}




\section{CONCLUSIONES}

Los genotipos evaluados mostraron una respuesta similar en cuanto a la duración de días hasta la floración; para la mayoría de los mismos se observó un hábito de crecimiento de arbusto indeterminado sin guía, el porte más bajo lo presentó la Tacarigua con 45,48 cm; mayor número de vainas por plantas la Sesentera y mayor peso de 100 semillas la Línea 13 con 28,9 g entre todas las variables para ambas localidades.

Los rendimientos en el estado de Barinas, fueron superiores para las variedades Balina con 1,10 t.ha- ${ }^{-1}$ Tacarigua con 1,06 t.ha ${ }^{-1} \mathrm{y}$ en estado de Monagas, la Línea 13 con 1,92 t.ha- ${ }^{-1}$, seguida de la Tacarigua con 1,68 t.ha ${ }^{-1}$ y Silvinera con 1,65 t.ha ${ }^{-1}$.

Para la variable rendimiento, resultó significativo el efecto genotipo y la localidad, donde SA 029 obtuvo el valor más bajo $\left(0,99\right.$ t.ha $\left.^{-1}\right)$ existiendo diferencias significativas entre este y el resto de los genotipos. La Tacarigua como variedad comercial mostró rendimientos estables para ambas localidades.

\section{RECOMENDACIONES}

Propiciar encuentros locales con los productores de la finca La Ceiba, sector Aparicio, municipio Piar, del estado Monagas y de la finca la Alborada, sector Mamon Mamonal, municipio Rojas, del estado Barinas, que generen intercambios para la divulgación de los resultados obtenidos y ensayos de fitomejoramiento participativo con vistas a definir los adecuados para cada ecosistema estudiado y se brinde una mayor información de calidad de las caraotas en función del gusto y preferencias.

\section{BIBLIOGRÁFIAS}

BARRIOS, A. y ORTEGA S. 1968. Resultados de ensayos de fertilización en caraota (Phaseolus vulgaris L.). Agronomía Tropical. 20(5):355 - 369.

BASTIDAS R, G. y CAMACHO. L. 1969. Competencia entre plantas y su efecto en el rendimiento y otras características del fríjol "caraota" (Phaseolus vulgaris L.). Acta Agronómica. 19(2):69 - 88.

BASTIDAS, G. 1974. Fríjol y caraota, producción, variedades y uso. ICA Informa. Santafé de Bogotá, Colombia. 9(12):3 - 06.

BENACCHIO, S. 1982. Algunas exigencias agroecológicas en 58 especies de cultivo con potencial de producción en el trópico americano - un compendio-. FONAIAP. Maracay. 1era Ed. CROMOTIP Caracas - Venezuela. $202 \mathrm{p}$.

CENTRO INTERNACIONAL DE AGRICULTURA TROPICAL (CIAT). 1984. Morfología de la planta de fríjol común. (Phaseolus vulagaris L.). Guía de estudio. $2^{\text {a }}$ Edición. Cali, Colombia. 49 p.

DEBOUCK, D. G. y R. HIDALGO.1985. Morfología de la planta de fríjol común. 16 - 24 p. En: Marcelino L., Fernández F. y Schoonhoven. Fríjol: Investigación y Producción. CIAT.

EXPÓSITO, R. P. y GARCÍA, B. N. 2010. Comportamiento productivo de cultivares de fríjol negro (Phaseolus vulgaris, L.) en la cooperativa de créditos y servicios "José Manuel Rodríguez" del municipio Jesús Menéndez. En: http://www.eumed.net/ cursecon/ecolat/cu/2011/epgb.htm. sp; consulta: febrero, 2013.

FIGUEROA, N. y WARNOCK, R. 2001. Estudio del crecimiento y desarrollo de la caraota (Phaseolus vulgaris L.) bajo diferentes densidades de población. III: Rendimiento y sus componentes. Revista de la Facultad de Agronomía. 27:105 - 117. 
INFOSTAT. 2002. Infostat versión 1.1 Manual del ususario. Grupo Infostat, FCA. Universidad Nacional de Córdoba. Primera Edición. Ed. Brujas. $61-90 p$.

INSTITUTO NACIONAL DE INNOVACIÓN AGRARIA (INIA). 2013. Red Agrometeorológica. Ministerio del Poder Popular para la Agricultura y Tierra. Instituto Nacional de Investigaciones Agrícolas de Venezuela. En: http://agrometeorologia.inia.gob.ve/index.php? option $=\mathrm{com}_{-}$ docman\&task=cat_view\&gid=158\&Itemid=31. 1p.; consulta: febrero, 2013.

INSTITUTO NICARAGUENSE DE TECNOLOGÌA AGROPECUARIA (INTA). 2004. Efecto de la aplicación de nitrógeno, fósforo y potasio para la producción de variedades de fríjol negro. INTA. Informe Anual Fríjol.

ISMAEL, S. N., WILLIAMS, H. M., REYES, W. W., y CORDÓN, E. 2011. Rendimiento de dos variedades mejoradas de fríjol, sembrados al voleo y al espeque, en Moss Pam, Waspam, rio Coco. Ciencia e interculturalidad. 9(2):112 - 127.

LOZADA, C., S. ORTEGA y N. SÁNCHEZ.1983. Comportamiento de 12 líneas de caraota (Phaseolus vulgaris L.) en Cubiro, estado Lara. Agronomía Tropical. 33(1-6):71 - 81.

MORROS, M. E. 2001. Cultivo de la caraota con énfasis en el estado Lara. Maracay, Venezuela. Instituto Nacional de Investigaciones Agrícolas. Centro de Investigaciones Agropecuarias del Estado Lara. (Serie D No. 2). 74 p.

NAJUL, C. y ANZALONE, A. 2006. Control de maleza con cobertura vegetal en el cultivo de caraota negra (Phaseolus vulgaris L.). Bioagro. 18(2):75 - 82.

ORGANIZACIÓN DE LAS NACIONES UNIDAS PARA LA ALIMENTACIÓN Y LA AGRÍCULTURA (FAO). 1980. Metodología de valoración económica.
En: http://www.fao.org/docrep/003/v8490s/ v8490s09.ht. 1p.; consulta: marzo, 2013.

ORTEGA, S., y BARRIOS, A. 2002. 'Tacarigua': Nueva variedad de caraota (Phaseolus vulgaris L.) Agronomía Tropical 22(4):435 - 438.

RODRÍGUEZ, E., GARCÍA, R. DE. y GONZÁLEZ, F. 1995. Poroto (Phaseolus vulgaris L.). Guía técnica para su cultivo. Ciudad de Panamá PROFIJOL. 27 p.

SCHARGEL, R. 1972. Características y génesis de una Cronosecuencia de suelos desarrollada sobre depósitos aluviales entre los ríos Boconó y Masparro, Estado Barinas. Agronomía Tropical. 22(4): 345 - 373.

SENASEM. 2010. Protocolo de evaluación de cultivares de leguminosas caraota (Phaseolus vulgaris L.) y fríjol (Vigna sp.) con fines de elegibilidad a certificación. 6 p.

SINGH. 2001. Broadening the genetic base of common beans cultivars: A review. Crop Science. 41:1659 $-1675$.

SNEDECOR, G. W., COCHRAN, W. G. y FULLER, J. R. 1984. Métodos estadísticos. Continental. México. $703 \mathrm{p}$.

ZINCK, A. y URRIOLA, P. L. 1971. Estudio edafológico. Valle Guarapiche. Estado Monagas. Ministerio de Obras Públicas (MOP). Barcelona, Venezuela.

VALDERRAMA, Y. N., NÚÑEZ, C. y DUARTE, A.1997. Evaluación de 26 genotipos de fríjol caraota (Phaseolus vulgaris L.) en la zona de Vega del Río Arauca. Agronomía Colombiana. 14(2):127 - 137.

VOYSEST, O. 2000. Mejoramiento genético del fríjol (Phaseolus vulgaris L.): legado de variedades de América Latina 1930-1999. CIAT. Cali, Colombia. 195 p. 